Series A

I. MATHEMATICA

590

\title{
THE FAMILY OF PDOL GROWTH-SETS IS PROPERLY INCLUDED IN THE FAMILY OF DOL GROWTH-SETS
}

BY

JUHANI KARHUMÄKI

H E L S I K I 1974

S U O M A A I N E T I E D E A K A T EMIA

doi:10.5186/aasfm. 1975.590 
Copyright (c) 1974 by

Academia Scientiarum Fennica

ISSN 0066-1953

ISBN 951.41.0207.X

Communicated 13 May 1974

KESKUSKIR.JAPAINO

HELSINKI 1974 


\section{Introduction}

L-systems have been introduced for biological purposes (see [3]). However, these have been studied intensively during the last few years from the formal language point of view (see [1] and [6]). A particularly interesting aspect within L-systems is the theory of growth functions. These have been studied for instance in [7] and [8].

The purpose of this note is to show that the family of PDOL growthsets is properly included in the family of DOL growth-sets. As a corollary of this result we also solve a problem introduced in [5], namely that $\mathscr{L}_{\mathrm{CPDOL}} \subsetneq \mathscr{L}_{\mathrm{CDOL}}$.

\section{Notations}

We assume that the reader is familiar with the standard formal language notations. For the definitions of DOL-systems, -languages, and-sequences we refer to [1]. We say that a DOL-system $G$ is $\lambda$-free, or a PDOL-system, iff there are no $\lambda$-productions in $G$.

If $G$ is a DOL-system, then $L(G)$ (resp. $E(G)$ ) means the language (resp. the sequence) generated by $G$. The growth-set generated by $G$ is

$$
|L(G)|=\{|P| \quad \mid P \in L(G)\},
$$

where $|P|$ means the length of the word $P$. Let

$$
E(G)=\omega_{0}, \omega_{1}, \ldots
$$

Then the growth-sequence generated by $G$ is

$$
|E(G)|=\left|\omega_{0}\right|,\left|\omega_{1}\right|, \ldots
$$

We say that a homomorphism $h: V_{1}^{*} \rightarrow V_{2}^{*}$ is a coding iff it maps each a letter to another letter. So all codings are length preserving.

A language $L$ is called a CDOL-language (resp. a CPDOL-language) iff there exists a DOL-system $G$ (resp. a PDOL-system $G$ ) and a coding $h$ such that

$$
L=h(L(G))
$$


The family of CDOL-languages (resp. CPDOL-languages) is denoted by $\mathscr{L}_{\text {CDOL }}$ (resp. $\mathscr{L}_{\text {CPDOL }}$ ).

\section{Lemmas}

We need the following three lemmas.

\section{Lemma 1. Let}

$$
|E(G)|=\left|\omega_{0}\right|,\left|\omega_{1}\right|, \ldots
$$

be a DOL growth-sequence. Then (1) is ultimately periodic modulo 2.

Proof. It is well known (see [7]), that, for $n \geq n_{0}$, (1) satisfies a recursion formula with integer coefficients, say

$$
\left|\omega_{n}\right|=\sum_{i=1}^{k} \alpha_{i}{ }^{\prime}\left(\omega_{n-i} \mid, \text { for } n \geq n_{0} .\right.
$$

Let $g$ be the canonical homomorphism of $Z$ onto $Z_{2}$. By applying $g$ to the above equation we obtain in the finite set $Z_{2}$ the recursion formula

$$
g\left(\left|\omega_{n}\right|\right)=\sum_{i=1}^{k} g\left(\alpha_{i}\right) g\left(\left|\omega_{n-i}\right|\right), \text { for } n \geq n_{0} .
$$

Thus, the sequence determined by this recursion formula must be ultimately periodic in $Z_{2}$. So we have proved Lemma 1 .

Let $H$ be the following DOL-system. The axiom is $a$, the alphabet is

$$
V=\left\{a, b, c, a_{1}, b_{1}, c_{1}, a_{2}, b_{2}, c_{2}\right\},
$$

and the productions are as follows:

$$
\begin{array}{lll}
a \rightarrow a_{1} a_{2}, & a_{1} \rightarrow a b c^{2}, & a_{2} \rightarrow \lambda, \\
b \rightarrow b_{1} b_{2}, & b_{1} \rightarrow b c^{2}, & b_{2} \rightarrow \lambda, \\
c \rightarrow c_{1} c_{2}, & c_{1} \rightarrow c, & c_{2} \rightarrow \lambda .
\end{array}
$$

Let

$$
L_{1}=\left\{P \in V^{*} \mid a \Rightarrow^{2 n} P, \text { for some } n \geq 0\right\}
$$

and

$$
L_{2}=\left\{P \in V^{*} \mid a \Rightarrow^{2 n+1} P, \text { for some } n \geq 0\right\} .
$$

Then the corresponding sequences are

$$
E_{1}=a, a b c^{2}, a b c^{2} b c^{4}, a b c^{2} b c^{4} b c^{6}, \ldots
$$


and

$$
E_{2}=a_{1} a_{2}, a_{1} a_{2} b_{1} b_{2}\left(c_{1} c_{2}\right)^{2}, \quad a_{1} a_{2} b_{1} b_{2}\left(c_{1} c_{2}\right)^{2} b_{1} b_{2}\left(c_{1} c_{2}\right)^{4}, \ldots
$$

So the language generated by $H$ is

$$
L(H)=L_{1} \cup L_{2}=\left\{a, a b c^{2} b c^{4} \ldots b c^{2 n} \mid n \geq 1\right\} \cup\left\{h(P) \mid P \in L_{1}\right\},
$$

where $h$ is the homomorphism of $\{a, b, c\}$ into $V$ defined by $h(y)=$ $y_{1} y_{2}$. Because

$$
1+n+2+4+\ldots+2 n=(n+1)^{2}
$$

the growth-set determined by $H$ is

$$
|L(H)|=\left\{n^{2}, 2 n^{2} \mid n \geq 1\right\} .
$$

We now put the elements of $|L(H)|$ in increasing order and let $X$ be this sequence. Denote

$$
X=x_{1}, x_{2}, \ldots
$$

Lemma 2. $X$ is not a DOL growth-sequence.

Proof. Assume the contrary: that a DOL-system $H_{1}$ generates the sequence $X$. Then, by Lemma $1,(2)$ is ultimately periodic modulo 2 . So there exist natural numbers $r$ and $s$ such that

$$
x_{r} \text { is an odd square }
$$

and for each $i$ and $j, i \geq 0,0 \leq j \leq s-1$,

$$
x_{r+j+i s} \equiv x_{r+j+(i+1) s}(\bmod 2) .
$$

Let $x_{r}=k^{2}$. Note that in (2) all odd integers are squares. So if $m$ is the number of odd integers in the period, then for each $i \geq 0$

$$
x_{r+i s}=(k+i 2 m)^{2} \text {. }
$$

But this implies that in each period there must also be a fixed number of integers of the form $2 n^{2}$. This however leads to a contradiction, as we shall now show.

For all $i \geq 0$, consider the natural numbers $z_{i}$ satisfying the condition

$$
(k+i 2 m)^{2}<2 z_{i}^{2}<(k+(i+1) 2 m)^{2},
$$

or equivalently,

$$
\frac{k}{\sqrt{2}}+i \sqrt{2} m<z_{i}<\frac{k}{\sqrt{2}}+i \sqrt{2} m+\sqrt{2} m .
$$

By what we have shown, the number of such $z_{i}^{\prime}$ s is the same for all $i \geq 0$. 
Trivially this number is either $[\sqrt{2} m]$ or $[\sqrt{2} m]+1$. Let $\delta_{0}$ and $\delta_{1}$ be positive real numbers defined by

$$
\delta_{0}=\sqrt{2} m-[\sqrt{2} m]
$$

and

$$
\delta_{1}=[\sqrt{2} m]+1-\sqrt{2} m .
$$

First, assume that for all $i \geq 0$ the number of $z_{i}^{\prime} \mathrm{s}$ is $[\sqrt{2} \mathrm{~m}]$. Choose $i_{0}$ such that $i_{0} \delta_{0}>1$. Then the length of the interval

$$
\left(\frac{k}{\sqrt{2}}, \frac{k}{\sqrt{2}}+i_{0} \sqrt{2} m\right)
$$

is

$$
i_{0} \sqrt{2} m=i_{0}[\sqrt{2} m]+i_{0} \delta_{0}>i_{0}[\sqrt{2} m]+1
$$

So the number of $z_{i}^{\prime}$ s in this interval is at least $i_{0}[\sqrt{2} m]+1$. On the other hand, by our assumption, their number is $i_{0}[\sqrt{2} \mathrm{~m}]$.

Secondly, assume that the number of $z_{i}^{\prime} \mathrm{s}$ is $i_{0}[\sqrt{2} m]+1$, for all $i \geq 0$. Now choose $i_{1}$ such that $i_{1} \delta_{1}>2$. Thus, the length of the interval

$$
\left(\frac{k}{\sqrt{2}}, \frac{k}{\sqrt{2}}+i_{1} \sqrt{2} m\right)
$$

$\mathrm{i}_{\mathrm{S}}$

$$
i_{1} \sqrt{2} m=i_{1}[\sqrt{2} m]+i_{1}-i_{1} \delta_{1}<i_{1}[\sqrt{2} m]+i_{1}-2
$$

Thus, in this interval there are at most $i_{1}[\sqrt{2} \mathrm{~m}]+i_{1}-1$ numbers $z_{i}$. But by our assumption, in this interval there must be $i_{1}[\sqrt{2} m]+i_{1}$ numbers of this kind.

Because both the cases lead to a contradiction, we have proved Lemma 2.

We also need the following lemma, which is Lemma 5.4. of [4].

Lemma 3. Let $G$ be a PDOL-system generating an infinite language. Then there exists a PDOL-system $G_{1}$ such that

(i) $|L(G)|=\left|L\left(G_{1}\right)\right|$,

(ii) the sequence $\left|E\left(G_{1}\right)\right|$ is strictly increasing.

\section{Results}

Now we are ready to establish our results.

Theorem 1. The family of PDOL growth-sets is a proper subset of the family of DOL growth-sets. 
Proof. The inclusion is trivial. It is proper because $|L(H)|$ is a DOL growth-set, but is not, by Lemmas 2 and 3, a PDOL growth-set.

As an immediate corollary of Theorem 1 we can solve a problem proposed in $[5]$.

Theorem 2. The family $\mathscr{L}_{\mathrm{CPDOL}}$ is properly included in the family $\mathscr{L}_{\text {CDOL }}$.

Examples of languages which lie in the difference $\mathscr{L}_{\mathrm{CDOL}} \backslash \mathscr{L}_{\mathrm{CPDOL}}$ are the languages $L(H)$ and $L=\left\{a^{n^{2}}, a^{2 n^{2}} \mid n \geq 1\right\}$.

We can generalize Theorem 1 to cover all growing DOL-systems, i.e., systems with an increasing growth-sequence. Of course any PDOL-system is a growing DOL-system.

Theorem 3. The family of growth-sets generated by growing DOL-systems is properly included in the family of DOL growth-sets.

Proof. It suffices to show that Lemma 3 can be generalized for growing DOL-systems. Assume that $G$ is a growing DOL-system with $E(G)=$ $\omega_{0}, \omega_{1}, \ldots$ In the following we use the notations of [4].

Let $M$ be the growth-matrix of $G, \pi$ the Parikh-vector associated with the axiom of $G$, and $\eta$ the column vector with all elements equal to 1 . Then the sequence

$$
d_{n}=\pi\left(M^{n}-M^{n-1}\right) \eta=\pi(M-I) M^{n-1} \eta, \quad n=1,2, \ldots
$$

tells us how much the length of the word grows during the $n$th step of the derivation. By (4), the $d_{n}^{\prime}$ s satisfy a recursion formula with integer coefficients. Thus, by the Theorem proved in [2], zeros occur in (4) ultimately periodically.

For all $i \geq 0$, let $\operatorname{Min}\left(\omega_{i}\right)$ denote the set of symbols occurring in $\omega_{i}$. It is well known that the sequence

$$
\operatorname{Min}\left(\omega_{0}\right), \operatorname{Min}\left(\omega_{1}\right), \ldots
$$

is ultimately periodic.

Consider now the sequence consisting of ordered pairs

$$
\left(s\left(d_{0}\right), \operatorname{Min}\left(\omega_{0}\right)\right),\left(s\left(d_{1}\right), \operatorname{Min}\left(\omega_{1}\right)\right), \ldots,
$$

where $s(0)=0$ and $s(n)=1$, for $n \geq 1$. Because the component sequences of (6) are ultimately periodic, so is the whole sequence (6).

From this point on the proof is a straightforward modification of the proof of Lemma 5.4. in [4] (it uses only the periodicity of (5)). We omit the details. 
Remark 1. The proof of Lemma 3 in [4] is constructive. However, our analogous proof for growing DOL-systems is not constructive, because we need the Theorem of [2].

Remark 2. In [4] M. Nielsen solves the growth-set equivalence problem for PDOL-systems by changing the considered PDOL-sequences effectively to strictly increasing PDOL-sequences (with the same growth-set). Lemma 2 shows that we cannot solve the growth-set equivalence problem for DOLsystems by this method. In fact, it is not known if this problem is decidable at all.

\section{Acknowledgement}

The author is grateful to Mr. M. Soittola for many helpful comments on this paper, and to Professor A. Salomaa for pointing out the problem.

Department of Mathematics,

University of Turku, Finland. 


\section{References}

[1] Herman, G. and Rozenberg, G.: Developmental systems and languages. - NorthHolland Publishing Company (to appear).

[2] LeCH, C.: A note on recurring series. - Arkiv Mat. 2 (1953), 417-421.

[3] Lindenmayer, A.: Mathematical models for cellular interactions in development. Parts I and II. - Journal of Theoretical Biology 18 (1968), 280-315.

[4] Nielsen, M.: On the decidability of some equivalence problems for DOL-systems. • Information and Control (to appear).

[5] -》- Rozenberg, G., SalomaA, A. and Skyum, S.: Nonterminals, homomorphisms and codings in different variations of OL-systems. Part I. Deterministic systems. Submitted for publication.

[6] Rozenberg, G. and SalomaA, A., (ed.): $L$-systems. - Springer Lecture Notes in Computer Science (to appear).

[7] SalomaA, A.: On exponential growth in Lindenmayer systems. - Indagationes Mathematicae 35 (1973), 23-30.

[8] Vitányi, P.: Structure of growth in Lindenmayer systems. - Indagationes Mathematicae 35 (1973), 247-253. 